\title{
Relative survival of patients with non-malignant central nervous system tumours: a descriptive study by the Austrian Brain Tumour Registry
}

A Woehrer ${ }^{\star}, 1$, M Hackl $^{2}$, T Waldhör ${ }^{3}$, S Weis ${ }^{4}$, J Pichler $^{5}$, A Olschowski $^{6}$, J Buchroithner $^{6}$, H Maier $^{7}$, G Stockhammer $^{8}$, C Thomé ${ }^{9}$, J Haybaeck ${ }^{10}$, F Payer ${ }^{11}$, G von Campe ${ }^{12}$, A Kiefer ${ }^{13}$, F Würtz ${ }^{13}$, G H Vince ${ }^{14}$, R Sedivy ${ }^{15}$, S Oberndorfer $^{16}$, $\mathrm{F}$ Marhold $^{17}$, K Bordihn ${ }^{18}$, W Stiglbauer ${ }^{19}$, U Gruber-Mösenbacher ${ }^{20}$, R Bauer ${ }^{21}$, J Feichtinger ${ }^{22}$, A Reiner-Concin ${ }^{23}$, W Grisold ${ }^{24}, \mathrm{C} \mathrm{Marosi}^{25}$, M Preusser ${ }^{25}$, K Dieckmann ${ }^{26}$, I Slavc ${ }^{27}$, B Gatterbauer ${ }^{28}$, G Widhalm ${ }^{28}$, C Haberler ${ }^{1}$ and J A Hainfellner ${ }^{1}$ on behalf of the Austrian Brain Tumour Registry

\begin{abstract}
${ }^{1}$ Institute of Neurology, Medical University of Vienna, Währinger Gürtel 18-20, A-1097 Vienna, Austria; ${ }^{2}$ Austrian National Cancer Registry, Statistics Austria, Guglgasse 13, A-1110 Vienna, Austria; ${ }^{3}$ Center for Public Health, Department of Epidemiology, Medical University of Vienna, Währinger Gürtel 18-20, A-1097 Vienna, Austria; ${ }^{4}$ Department of Pathology and Neuropathology, State Neuropsychiatric Hospital Wagner-Jauregg, Linz, Wagner-Jauregg-Weg 15, A-4020 Linz, Austria; ${ }^{5}$ Internal Medicine and Neurooncology, State Neuropsychiatric Hospital Wagner-Jauregg, Wagner-Jauregg-Weg 15, A-4020 Linz, Austria; ' $D$ Department of Neurosurgery, State Neuropsychiatric Hospital Wagner-Jauregg, Wagner-Jauregg-Weg 15, A-4020 Linz, Austria; ${ }^{7}$ Department of Neuropathology, Institute of Pathology, Medical University of Innsbruck, Christoph-Probst-Platz Innrain 52, A-6020 Innsbruck, Austria; ${ }^{8}$ Department of Neurology, Medical University of Innsbruck, Christoph-Probst-Platz Innrain 52, A-6020 Innsbruck, Austria; 'Department of Neurosurgery, Medical University of Innsbruck, Christoph-Probst-Platz Innrain 52, A-6020 Innsbruck, Austria; ${ }^{10}$ Department of Neuropathology, Institute of Pathology, Medical University of Graz, Auenbruggerplatz 25, A-8036 Graz, Austria; ${ }^{11}$ Division of General Neurology and Division of Neuroradiology, Medical University of Graz, Auenbruggerplatz 25, A-8036 Graz, Austria; ${ }^{12}$ Department of Neurosurgery, Medical University of Graz, Auenbruggerplatz 25, A-8036 Graz, Austria; ${ }^{13}$ Institute of Pathology, State Hospital Klagenfurt, St Veiter Strasse 47, A-9020 Klagenfurt, Austria; ${ }^{14}$ Department of Neurosurgery, State Hospital Klagenfurt, St Veiter Strasse 47, A-9020 Klagenfurt, Austria; ${ }^{15}$ Department of Clinical Pathology, General Hospital St Pölten, Probst-FührerStrasse 4, A-3100 St Pölten, Austria; ${ }^{16}$ Department of Neurology, General Hospital St Pölten, Probst-Führer-Strasse 4, A-3100 St Pölten, Austria; ${ }^{17}$ Department of Neurosurgery, General Hospital St Pölten, Probst-Führer-Strasse 4, A-3100 St Pölten, Austria; ${ }^{18}$ Department of Neurosurgery, Christian Doppler Clinic, Paracelsus Private Medical University, Strubergasse 21, A-5020 Salzburg, Austria; ${ }^{19}$ Institute of Pathology, General Hospital Wiener Neustadt, Corvinusring 3-5, A-2700 Wiener Neustadt, Austria; ${ }^{20}$ Department of Pathology, Feldkirch State Hospital, Carinagasse 47, A-6807 Feldkirch, Austria; ${ }^{21}$ Department of Neurosurgery, Feldkirch State Hospital, Carinagasse 47, A-6807 Feldkirch, Austria; ${ }^{22}$ Department of Pathology, Krankenanstalt Rudolfstiftung, Juchgasse 25, A-1030 Vienna, Austria; ${ }^{23}$ Institute of Pathology, Danube Hospital, Langobardenstrasse 122, A-1220 Vienna, Austria; ${ }^{24}$ Department of Neurology, KFJ-Hospital Vienna, Kundratstrasse 3, A-1100 Vienna, Austria; ${ }^{25}$ Department of Medicine I, Medical University of Vienna, Währinger Gürtel 18-20, A-1097 Vienna, Austria; ${ }^{26}$ Department of Radiation Oncology, Medical University of Vienna, Währinger Gürtel 18-20, A-1097 Vienna, Austria; ${ }^{27}$ Department of Paediatrics, Medical University of Vienna, Währinger Gürtel 18-20, A-1097 Vienna, Austria and ${ }^{28}$ Department of Neurosurgery, Medical University of Vienna, Währinger Gürtel 18-20, A-1097 Vienna, Austria
\end{abstract}

Background: Unlike malignant primary central nervous system (CNS) tumours outcome data on non-malignant CNS tumours are scarce. For patients diagnosed from 1996 to 2002 5-year relative survival of only $85.0 \%$ has been reported. We investigated this rate in a contemporary patient cohort to update information on survival.

Methods: We followed a cohort of 3983 cases within the Austrian Brain Tumour Registry. All patients were newly diagnosed from 2005 to 2010 with a histologically confirmed non-malignant CNS tumour. Vital status, cause of death, and population life tables were obtained by 31 December 2011 to calculate relative survival.

Results: Overall 5-year relative survival was 96.1\% (95\% Cl 95.1-97.1\%), being significantly lower in tumours of borderline (90.2\%, 87.2-92.7\%) than benign behaviour (97.4\%, 96.3-98.3\%). Benign tumour survival ranged from 86.8 for neurofibroma to $99.7 \%$ for Schwannoma; for borderline tumours survival rates varied from 83.2 for haemangiopericytoma to $98.4 \%$ for myxopapillary ependymoma. Cause of death was directly attributed to the CNS tumour in $39.6 \%$, followed by other cancer (20.4\%) and cardiovascular disease (15.8\%).

Conclusion: The overall excess mortality in patients with non-malignant CNS tumours is $5.5 \%$, indicating a significant improvement in survival over the last decade. Still, the remaining adverse impact on survival underpins the importance of systematic registration of these tumours.

*Correspondence: A Woehrer; E-mail: adelheid.woehrer@meduniwien.ac.at

Received 24 July 2013; revised 4 October 2013; accepted 21 October 2013; published online 19 November 2013

(c) 2014 Cancer Research UK. All rights reserved 0007-0920/14 
Primary central nervous system (CNS) tumours comprise a large spectrum of distinct entities that differ substantially in terms of histomorphology, tumour biology, and behaviour. According to the third edition of the International Classification of Diseases for Oncology (ICD-O) tumours of benign, borderline, and malignant behaviour are distinguished (Fritz et al, 2000). While malignant tumours, that is, 'cancer' (ICD-O3/3) are characterised by the invasion of surrounding tissues and their potential for metastatic spread, benign (ICD-O3/0) and borderline (ICD-O3/1) tumours show restricted local growth without the potential for spread (Fritz et al, 2000). Thus, benign and borderline (=non-malignant) tumours are generally considered curable by resection only and usually do not require adjuvant treatment.

Non-malignant CNS tumours differ from their systemic counterparts with regard to their exclusive location within the CNS rendering them close to eloquent areas. The potential for malignant transformation of some benign tumour types (Evans et al, 2006), the occurrence of multiple tumours in the case of genetic tumour syndromes as well as unexpected events in the perioperative course of neurosurgerical procedures (Cornwell et al, 2012; Solheim et al, 2012) constitute further factors that have an impact on the functional outcome and quality of life of the affected patients. Nevertheless, in contrast to malignant CNS tumours, non-malignant tumours are not consistently reported through cancer registries, thus the population-based experience with these tumours is still limited (McCarthy et al, 2009).

Following an initiative of the Central Brain Tumour Registry of the United States (CBTRUS) the registration of non-malignant CNS tumours has become legally mandatory in the United States in 2004 (McCarthy et al, 2013). Similarly, selected cancer registries across the Scandinavian countries, the United Kingdom, Japan, and Israel (Sant et al, 2012; Shibui, 2012; Israel National Cancer Registray, 2013) as well as specialized brain tumour registries emerging in France and Austria (Bauchet et al, 2007; Woehrer 2013) are reporting incidence data on non-malignant CNS tumours, which account for $\sim 50 \%$ of all primary CNS tumours (Wohrer et al, 2009; Dolecek et al, 2012). Yet, with regard to the outcome of these patients population-based data are even scarcer. Probably, the most comprehensive effort, so far, has come from the Eurocare project, which has compiled cancer registry data across different European regions to evaluate patient survival in a pan-European context (Sant et al, 2012). Their recent work also included survival data of patients with non-malignant CNS tumours, where they found a 5-year relative survival rate of only $85.0 \%$, thus indicating an alarming excess risk of death (Sant et al, 2012). In addition, they observed substantial regional variations with poorest outcome in Eastern Europe (Sant et al, 2012). However, the survival analysis was based on a patient cohort diagnosed from 1996 to 2002. Thus, it does not yet reflect the beneficial impact of innovations in the field of neurooncology, which have taken place over the last decade. New diagnostic standards (Louis et al, 2007), widely available sophisticated functional imaging techniques (Kuhnt et al, 2013) as well as improved neurosurgical techniques (Chen et al, 2011; Kuhnt et al, 2012) and perioperative patient management (Wong et al, 2012) are among the major factors. As up-to-date information on survival serves as an important reference for clinicians and scientists (Talback et al, 2004), we readdressed and further scrutinised this issue in a large contemporary cohort of patients with non-malignant CNS tumours diagnosed from 2005 to 2010.

\section{MATERIALS AND METHODS}

A consecutive cohort of patients with non-malignant CNS tumours (ICD-O3 behaviour codes/0 benign and/1 borderline) was retrieved from the records of the Austrian Brain Tumour Registry
(ABTR, $n=3983$ ). Austrian Brain Tumour Registry scientific activities have been approved by the local ethics committee and data protection authorities (approval no 550/2005). Persons without permanent Austrian residency were excluded. All cases were newly diagnosed in the time period 2005-2010 across all Austrian neuropathology departments $(n=11)$, and had a histologically confirmed diagnosis of a non-malignant CNS tumour. Reported patient parameters included personal identifiers, gender, date of birth, age at diagnosis, histopathological diagnosis, and tumour location. In cooperation with Statistics Austria, the vital status of the patient cohort (Austrian National Cancer Registry, Statistics Austria) as well as life tables of the Austrian population (Social Statistics, Statistics Austria) were obtained with a last up-date by 31 December 2011. In addition, information on cause-specific death according to the International Statistical Classification of Diseases and Related Health Problems (ICD-10) was available from death certificates (Causes of Death Statistics, Statistics Austria). Cause-specific deaths were grouped into the following reasonable categories: CNS tumour, other cancer, cardiovascular disease, infection, metabolic disease, other CNSrelated causes (including CNS trauma and haemorrhage but also dementias), and other non-CNS-related causes.

All statistical analyses were performed using Microsoft Excel, SAS (http://www.pauldickman.com), and SPSS software packages. Group comparisons were assessed with $t$ - and $\chi^{2}$ tests. In order to eliminate the effect of competing causes of mortality relative survival rates were calculated. Relative survival is defined as the ratio of the observed survival in the patient group and the expected survival in a sex- and age-matched disease-free population (Ederer et al, 1961). Life table methods were applied to estimate observed survival, and the Ederer II model (Ederer and Heise, 1959) was applied to estimate expected survival using calendar year-, sex-, and age-specific life tables provided by Statistics Austria. In order to warrant comparability with clinical studies overall survival rates are provided along with the relative survival rates, where appropriate. Overall and relative survival rates are not displayed for tumours with less than 10 observations during the study period. Subgroup analyses were performed for gender, tumour location (ICD-O3 topography codes) (Fritz et al, 2000), and selected age cohorts $(0-18,19-44,45-59,60-74$, and 75 years +$)$. Substratifications of tumour type according to gender and age as well as tumour behaviour according to age were performed for tumours with adequately large sample sizes. Confidence intervals are not displayed in the case of insufficient sample size (referred to as asterisk). The study is retrospective and descriptive in nature. $P$-values and confidence intervals should therefore be interpreted as explorative only. No adjustments for multiple tests were applied.

\section{RESULTS}

Patient characteristics. The total cohort of non-malignant CNS tumours consisted of 3983 cases, among which 83.0\% $(n=3305)$ constituted tumours of benign and 17.0\% $(n=678)$ tumours of borderline behaviour. Within the group of benign brain tumours, females were more commonly affected than males (F/M ratio 1.8), whereas no major gender difference was observed for the group of borderline tumours (F/M ratio 1.1). A detailed distribution of tumour entities per category is provided in Table 1. Among the group of benign CNS tumours, the most common entities were meningioma WHO grade I $(n=1,914,57.9 \%)$, pituitary adenoma $(n=757,22.9 \%)$, and Schwannoma $(n=544,16.5 \%)$, whereas meningioma WHO grade II $(n=235,34.7 \%)$, pilocytic astrocytoma ( $n=109,16.1 \%)$, and haemangioblastoma ( $n=76,11.2 \%)$ constituted the most common tumours of borderline behaviour. Gender distribution was approximately equal for the majority of 
tumour types except benign meningioma (WHO grade I), which showed a 2.9-fold female excess. Similarly, subependymoma and myxopapillary ependymoma tended to be more common in females $(\mathrm{M} / \mathrm{F}$ ratios of 0.6 and 0.7 , respectively), whereas central neurocytoma, neurofibroma, dysembryoplastic neuroepithelial tumour, and pilocytic astrocytoma showed a male preference (M/F ratios of $2.7,1.4,1.4$, and 1.3 , respectively). Overall, median age at diagnosis was significantly higher among patients with benign CNS tumours (57.2 years, range 3.6 months-92.0 years) compared with tumours of borderline behaviour (47.0 years, range 4.8 months -84.6 years; $P<0.0001$ ) but clearly varied according to tumour type (see Table 1) from pilocytic astrocytoma (median age at onset 14.1 years) to meningioma WHO grade II (60.3 years). With regard to tumour topography, $54.0 \%$ of tumours were located within the meninges, $19.1 \%$ within the pituitary gland, $14.5 \%$ occurred within cranial nerves, and $10.2 \%$ within the CNS parenchyma.

Death-specific mortality. Of the total cohort, $10.5 \%(n=417)$ deceased during the observation period. The fraction was significantly higher among patients with CNS tumours of borderline behaviour (15.5\%) compared with benign tumour types $(9.4 \%$; $P<0.0001)$. According to death certificates, in $39.6 \%$ of the patients the death was directly attributed to the CNS tumour (34.9\% of deaths from benign tumours, $53.3 \%$ of deaths from tumours of borderline behaviour, respectively, see Figure 1).
Common competing causes of death included other cancer $(20.4 \%)$ and cardiovascular disease $(15.8 \%)$, which were both more prevalent among benign CNS tumour patients $(22.8 \%$ and $19.6 \%$, compared with $13.3 \%$ and $4.8 \%$, respectively). The most common other type of cancer was lung cancer, which constituted $21.2 \%$ (18 persons) of all cancer-related deaths, whereas other cancer types accounted for less than 5\% (4 individuals) each. The time interval from neurosurgical intervention to cause-specific death was shortest for metabolic disease (median 4 months) and infections (median 6.5 months) ranging up to 34.7 months for cardiovascular disease. Central nervous system tumour-related death occurred on average at 16.0 months after diagnosis (s.d. 24.2 months, ranging from 0 to 89.0 months). Detailed information on causes of death and median time to death is provided for brain tumour types with more than five events of death in Table 2. Median CNS-related tumour death was shortest, that is, 1 month after diagnosis, for pituitary adenoma and haemangioblastoma patients.

Relative survival. One- and five-year cumulative relative survival rates (cRSR) for patients with non-malignant CNS tumours are presented in Table 3 . There was a small but statistically significant excess mortality in patients with non-malignant CNS tumours compared with the general population. The relative survival rates were significantly lower in patients with tumours of borderline as




compared with benign behaviour (see also Figure 2). Within behavioural categories, male performed worse compared with female patients, although the gender difference in rates - based on overlapping confidence intervals - did not reach statistical significance.

Tumour type-specific 1- and 5-year cRSR are listed in Table 4. Compared with the general population, all tumour types together showed impaired survival. Whereas some tumour types showed a small but not significant restriction in survival, for example, pituitary adenoma, Schwannoma (WHO grade I), and ganglioglioma (WHO grade I/II), the following tumours demonstrated a statistically significant impaired relative survival throughout the entire observation period: meningioma WHO grades I and II, dysembryoplastic neuroepithelial tumour, neurofibroma, pilocytic astrocytoma, haemangioblastoma, craniopharyngioma, haemangiopericytoma, subependymoma, and central neurocytoma. With regard to benign meningioma subtype analysis revealed that impaired survival was enhanced in patients with meningothelial meningiomas in contrast to other subtypes (fibrous, psammomatous, transitional, angiomatous, metaplastic, and microcystic Figure 3). In several tumour types including meningioma a trend towards worse outcome of male patients was noted, although which did not reach statistical significance (Table 4).

Stratification into age cohorts showed worse outcome with increasing age, overall and for individual tumour types (Figure 4). The youngest age cohort (0-18 years, 169 individuals) had a slightly worse outcome as compared with the 19-44 years age cohort (881 individuals); however, this difference was statistically not significant. Among patients above age 75 years an early drop of the survival rate was seen, which was followed by a secondary incline. This dynamic in the rate of elderly patients was mainly due to benign meningioma patients.

Although a considerable fraction of tumours (41.3\%) had imprecisely defined topography codes (i.e., C70.9 meninges, not otherwise specified NOS; C72.5 cranial nerve, NOS; C71.0 cerebrum, NOS) (Fritz et al, 2000), cRSR demonstrated impaired survival for tumours of the cerebral meninges, optic nerve, craniopharyngeal duct, brain stem, cerebellum, and ventricles (see Table 5). Subgroup analysis according to tumour location showed that patients with pilocytic astrocytomas of the optic tract/ chiasm $(n=15)$ had worse outcome (5-year cRSR 86.9\% (56.5-96.7\%)) compared with cerebellar tumours $(n=32$; 5-year cRSR $100.4 \%$ (CI contains 1)). Likewise, patients with cerebral meningiomas $(n=714)$ performed worse (5-year cRSR 95.2\% (92.5-97.4\%)) compared with their spinal counterparts $(n=93$; 5 -year cRSR 99.0\% (89.3-104.8\%)). In contrast, the rate for acoustic Schwannoma $(n=195$, 5-year cRSR $100.4 \%$ (95.9-102.7\%)) corresponded to that for Schwannomas at all other sites $(n=312,5$-year cRSR 98.7\% (95.2-101.0\%)).

\section{DISCUSSION}

In contrast to malignant CNS tumours, outcome data on nonmalignant CNS tumours are scarce. This is mainly due to the fact that these tumours are generally not considered as 'cancer' and thus, are frequently not systematically registered (Fritz et al, 2000). Outcome analyses for these types of tumours are further hampered as affected individuals are usually not supposed to die due to their CNS tumour but rather due to competing causes of death. This was also an important finding of the present study. Common competing causes of death included cancer and cardiovascular disease, which were both more prevalent among patients with benign than borderline CNS tumours, most likely reflecting the older age at disease onset of patients with benign tumours and the higher prevalence of those diseases with increasing age. In contrast, deaths from metabolic disease (including hepatic and renal failure) and infections occurred relatively early following neurosurgical interventions. These early events might indeed comprise a small fraction of patients, who experience perioperative mortality. Yet, detailed information on the hospital records or autopsy reports of these patients has not been available, and thus the exact prevalence of perioperative mortality remains unclear. Interestingly, however, $\sim 40 \%$ of all events of death were directly attributed to the non-malignant CNS tumour. Among the latter median time to death was strikingly short for pituitary adenoma and haemangioblastoma patients - a finding that needs to be cautiously interpreted in the light of small sample sizes but clearly deserves further attention.

Still, the information of cause-specific death has been obtained from death certificates, which carry the inherent problem of considerable inter-rater variability across different centers and practitioners (Burger et al, 2012; Sutra et al, 2012; Hu et al, 2013; Lafrance et al, 2013). Thus, cause-specific mortality from death certificates cannot generally be considered as objective outcome measure. Therefore, in order to assess any excess mortality in patients with non-malignant CNS tumours compared with the general population, we calculated relative survival rates, which are considered as gold standard for cause-specific survival analyses. Compared with baseline data from the Eurocare project (5-year RSR from non-malignant brain tumours of only $85.0 \%$ based on patients diagnosed from 1996 to 2002) (Sant et al, 2012), we found a considerably higher survival rate of up to $96.1 \%$ in our large contemporary patient series. The difference in rates might be even slightly enhanced considering the fact that Sant et al (2012) used the Hakulinen model to estimate relative survival, which yields comparable results but may lead to overestimation of rates in presence of many censored cases (Cho et al, 2011). Nevertheless, the observed increase confirms a trend, which has already been present in the Eurocare data (Sant et al, 2012) and may partly correspond to earlier and refined diagnostic procedures including improved and more widely available neuroimaging techniques (Legler et al, 1999; Klaeboe et al, 2005; Sant et al, 2012). Furthermore, a high health status of the Austrian general population and/or a lower threshold to undergo CT and MRI scans for minor complaints need to be taken into account when comparing Austria with a pan-European study which includes less favourable Eastern European countries.

All patients included in this study were diagnosed in specialized Austrian neurooncology centers by neurosurgical resection from 2005 to 2010 and had a histologically confirmed non-malignant CNS tumour, thereby warranting direct comparability with Eurocare data. Although a central histopathology review was not feasible for the present study, all cases were diagnosed by experienced board-certified neuropathologists using WHO consensus criteria for tumour typing, thereby warranting high diagnostic standards across Austria. Information on persons, who did not undergo neurosurgical resection (watchful waiting) due to a number of reasons including poor health condition at perioperative evaluation, are not yet routinely available through ABTR (Wohrer et al, 2009). Thus, the herein observed rates might be biased towards optimistic survival, as patients with poor preoperative performance scores were likely to be not included. According to internationally available data, the majority of cancer registries including also cancer registries with experience in reporting benign CNS tumours, report a rate of microscopically verified CNS tumours of more than $85 \%$ (Sant et al, 2012). Still, the exact prevalence of non-malignant CNS tumour patients, who are on a wait-and-see strategy for various reasons (asymptomatic patients, stable disease, poor performance status, neoadjuvant chemo- or radiotherapy) is largely unknown and more efforts have to be undertaken to register these patients systematically. 


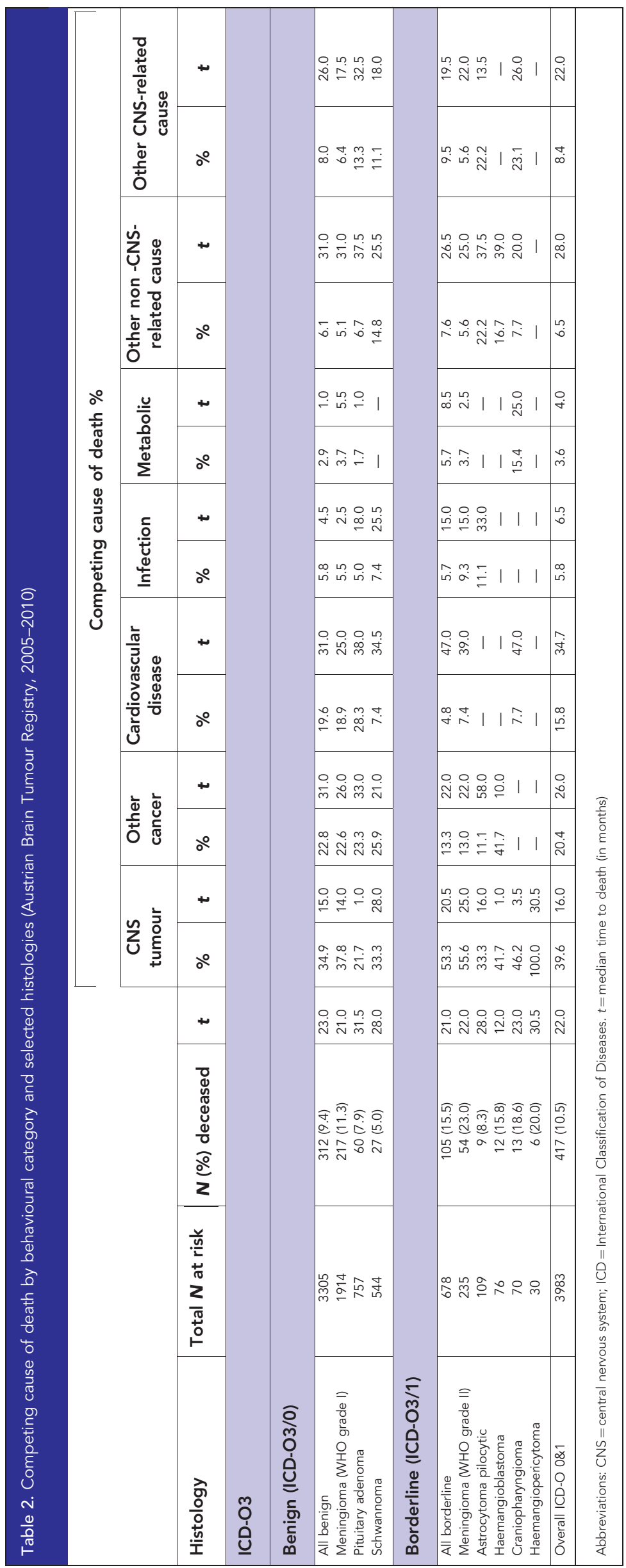


Table 3. Cumulative relative and overall survival rates for non-malignant CNS tumours according to ICD-O3 behaviour codes (Austrian Brain Tumour Registry, 2005-2010)

\begin{tabular}{|c|c|c|c|c|c|c|}
\hline ICD-O3 behaviour & $\mathbf{N}(\%)$ & 1-year cRSR (95\% Cl) & 1-year cOS $(95 \% \mathrm{Cl})$ & $\mathbf{N}(\%)$ & 5-year cRSR (95\% Cl) & 5 -year cOS $(95 \% \mathrm{Cl})$ \\
\hline \multicolumn{7}{|c|}{ ICD-O3/0\&1 non-malignant } \\
\hline $\begin{array}{l}\text { Overall } \\
\text { Male } \\
\text { Female } \\
\end{array}$ & $\begin{array}{l}3983(100.0) \\
1528(100.0) \\
2455(100.0)\end{array}$ & $\begin{array}{l}97.3(96.7-97.9) \\
97.1(95.9-98.0) \\
97.5(96.7-98.2)\end{array}$ & $\begin{array}{l}96.3(95.8-96.8) \\
95.7(94.6-96.6) \\
96.7(95.9-97.3)\end{array}$ & $\begin{array}{l}3194(80.2) \\
1194(78.1) \\
2000(81.5)\end{array}$ & $\begin{array}{l}96.1(95.1-97.1) \\
94.7(92.8-96.4) \\
97.0(95.8-98.0)\end{array}$ & $\begin{array}{l}90.6(90.1-91.1) \\
88.1(86.3-89.6) \\
92.2(91.1-93.2)\end{array}$ \\
\hline \multicolumn{7}{|l|}{ ICD-O3/0 benign } \\
\hline $\begin{array}{l}\text { Overall } \\
\text { Male } \\
\text { Female }\end{array}$ & $\begin{array}{l}3305(100.0) \\
1200(100.0) \\
2105(100.0)\end{array}$ & $\begin{array}{l}97.8(97.1-98.3) \\
97.4(96.1-98.4) \\
98.0(97.1-98.6)\end{array}$ & $\begin{array}{l}96.7(96.0-97.2) \\
96.0(94.7-97.0) \\
97.1(96.2-97.7)\end{array}$ & \begin{tabular}{|r|}
$2660(80.5)$ \\
$949(79.1)$ \\
$1.711(81.3)$
\end{tabular} & $\begin{array}{l}97.4(96.3-98.3) \\
96.4(94.3-98.2) \\
97.9(96.6-99.0)\end{array}$ & $\begin{array}{l}91.5(90.4-92.4) \\
89.2(87.3-90.9) \\
92.8(91.6-93.9)\end{array}$ \\
\hline \multicolumn{7}{|l|}{ ICD-O3/1 borderline } \\
\hline $\begin{array}{l}\text { Overall } \\
\text { Male } \\
\text { Female }\end{array}$ & $\begin{array}{l}678(100.0) \\
328(100.0) \\
350(100.0)\end{array}$ & $\begin{array}{l}95.3(93.3-96.8) \\
95.8(92.8-97.8) \\
94.8(91.8-96.8)\end{array}$ & $\begin{array}{l}94.5(92.5-96.0) \\
94.8(91.8-96.7) \\
94.3(91.3-96.3)\end{array}$ & $\begin{array}{l}534(78.8) \\
245(74.7) \\
289(82.6)\end{array}$ & $\begin{array}{l}90.2(87.2-92.7) \\
88.6(83.8-92.4) \\
91.7(87.6-94.7)\end{array}$ & $\begin{array}{l}86.4(83.5-88.8) \\
83.9(97.4-87.5) \\
88.8(84.9-91.7)\end{array}$ \\
\hline
\end{tabular}
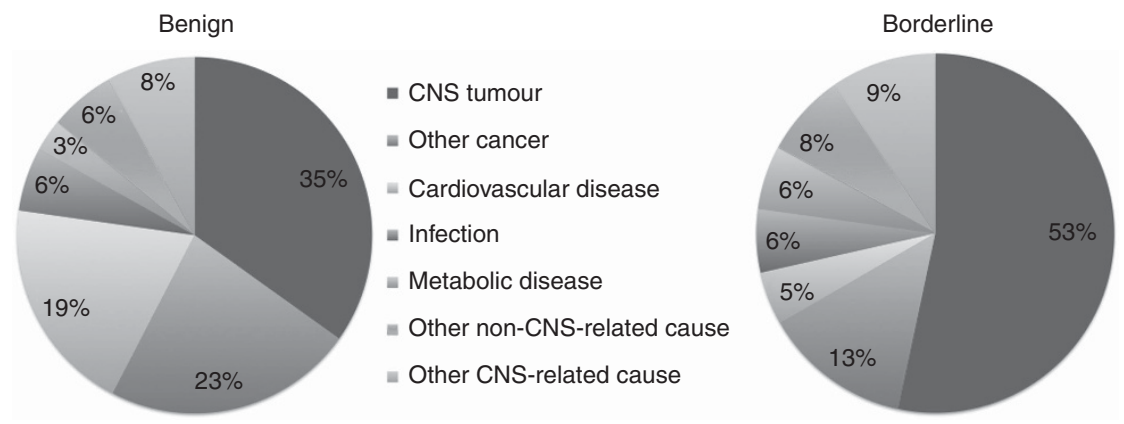

Figure 1. Competing causes of death among patients with benign and borderline CNS tumours (ABTR, 2005-2010). Cause of death in both categories was most commonly directly attributed to the CNS tumour. The fraction was higher among borderline tumours as compared with benign CNS tumours. Common competing causes of death included other cancer and cardiovascular disease, which were more prevalent among benign CNS tumour patients.

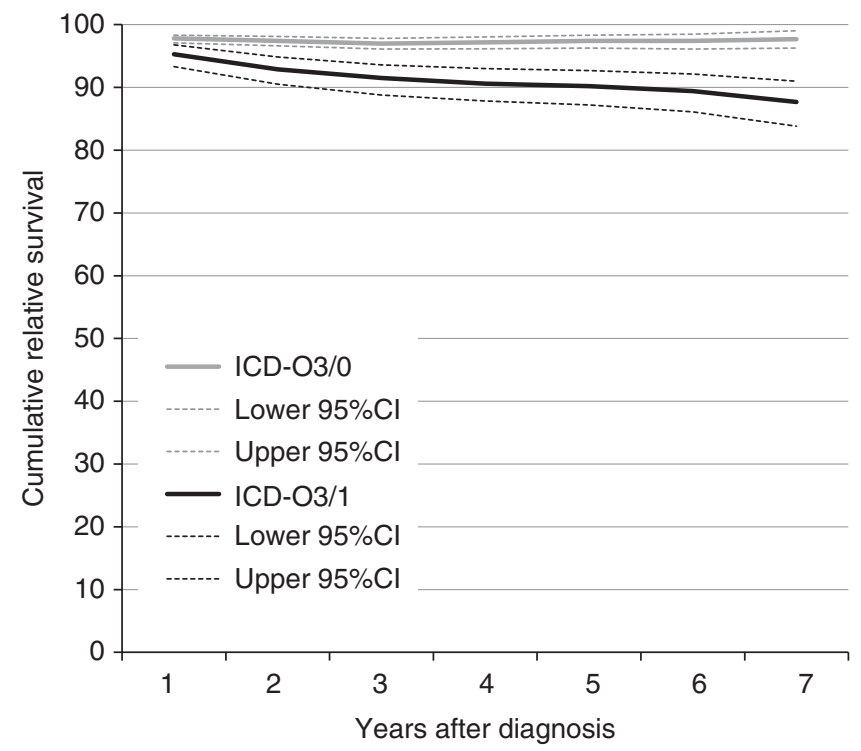

Figure 2. Cumulative relative survival rates of patients with nonmalignant CNS tumours over time according to ICD-O3 behaviour codes (ABTR, 2005-2010). Relative survival is inferior in patients with tumours of borderline behaviour (ICD-O3/1) as compared with benign behaviour (ICD-O3/0).
Although neurosurgical resection is considered the standard of care for the majority of non-malignant CNS tumour types, there is also a role for radio- or chemotherapy, hormonal therapy, and stereotactic radiosurgery in the treatment of certain tumour types, for example, pilocytic astrocytoma, subtypes of pituitary adenoma, or atypical meningioma (Zachenhofer et al, 2006; Gnekow et al, 2012; Vroonen et al, 2012; Coskun et al, 2013). Thus, detailed information on the outcome of specific tumour entities instead of presenting summary rates on groups of tumours is crucial. This is probably the most relevant aspect of the present study, which covers a huge portfolio of specific non-malignant CNS tumour entities. Herein, survival is first separately assessed for benign and borderline CNS tumours. As expected, lower relative survival rates were observed for tumours of borderline as compared with benign behaviour, reflecting the more aggressive disease course of the former. In line with the Eurocare data, survival decreased with increasing age at diagnosis (Sant et al, 2012).

Tumours with least favourable outcome included neurofibroma, craniopharyngioma, and haemangiopericytoma with excess mortalities of above $10 \%$. On the other end of the spectrum were patients with benign meningioma WHO grade I, Schwannoma, and pituitary adenoma, whose favourable outcomes showed almost no difference to the general population. The most common tumour types in our series included meningioma, pituitary adenoma, Schwannoma, and pilocytic astrocytoma. 


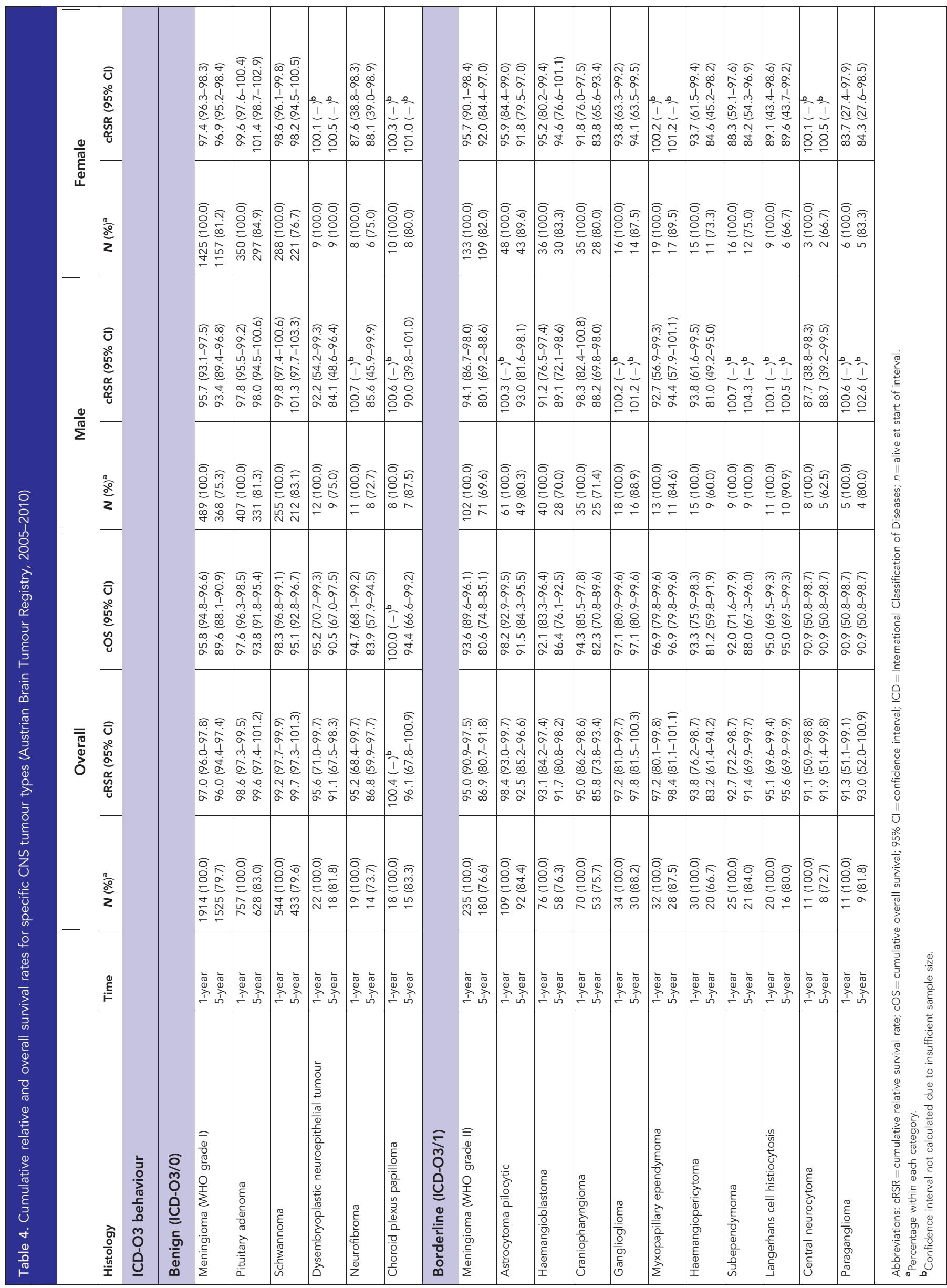




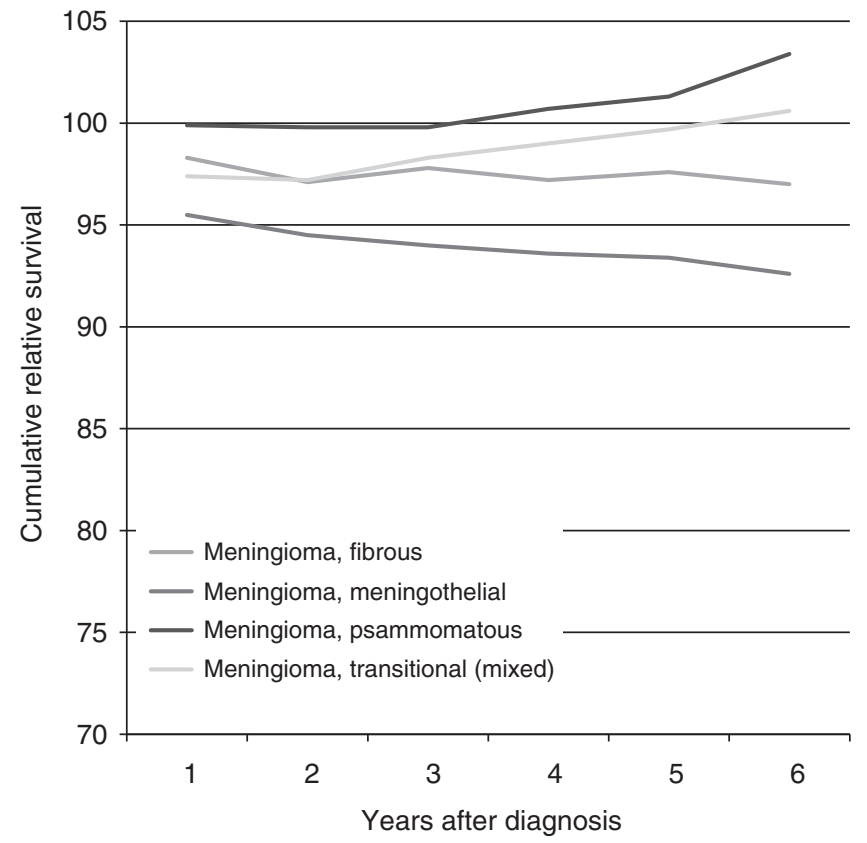

Figure 3. Cumulative relative survival rates for WHO grade I meningioma patients according to histopathological subtype (ABTR, 2005-2010). Patients with meningothelial subtype meningiomas show worse outcome as compared with other common meningioma subtypes (difference not statistically significant).

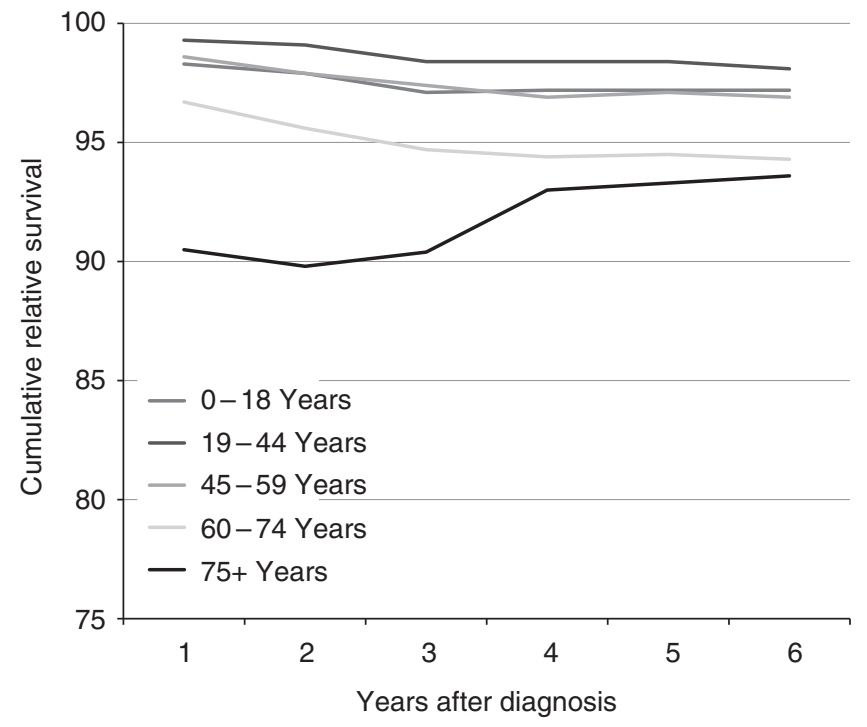

Figure 4. Cumulative relative survival rates of patients with nonmalignant CNS tumours according to age cohorts (ABTR, 2005-2010). Subgroup analysis according to age cohorts shows decreasing survival with increasing age. In elderly patients (75 + years) an early drop of the rate is present, which is followed by a secondary incline. This pattern is not seen in other age cohorts.

With regard to benign meningioma (WHO grade I) impaired survival has been previously implicated by data of the Swedish Cancer Registry, based on a patient cohort diagnosed from 1960 to 1998 (Talback et al, 2004). Similarly, Eurocare found a 5-year relative survival for meningioma of $88.7 \%$, ranging from $79.5 \%$ in Eastern Europe to $93.4 \%$ in Northern Europe (Sant et al, 2012). However, their data included both benign and borderline meningioma variants. Considering only benign meningioma, a large, retrospective analysis of 205 patients from 1985 to 2003 found 5-year relative survival rates of $92 \%$, which is slightly below the herein observed rate (van Alkemade et al, 2012). Of note, by reporting longer follow-up times (exceeding 5 years), they were able to demonstrate a prolonged continuous decline in survival, indicating a significant long-term mortality from these tumours. However, neither Eurocare data nor the study by van Alkemade et al (2012) observed gender-specific differences in survival, which contrasts the herein observed trend towards worse outcome for male patients. Improved survival of females has already been described for many cancers and has been attributed to fewer comorbidities and higher clinical performance of females (Cook et al, 2009; Sant et al, 2012). As ABTR data are based on accurate histopathological diagnoses, our data first allowed for subgroup analysis of histopathological meningioma subtypes, and interestingly, we observed increased excess mortality for patients with meningothelial meningiomas. The reason for this observation remains obscure on basis of our data. One possible explanation might be a higher likelihood of this meningioma variant for infiltration of surrounding tissues, for example, the skull base.

Atypical meningiomas differ from benign meningiomas in several aspects including their lower incidence, little if any gender predominance, and a considerably worse prognosis, which lies between benign and anaplastic meningiomas (Louis et al, 2007). They carry a two-fold increased risk of death at 3-5 years (Perry et al, 2004; Louis et al, 2007). Single studies based on small cohorts of 17-42 patients diagnosed in the 1940-90s, have reported 5-year overall survival rates of 91-95\% (Palma et al, 1997; Goyal et al, 2000). Interestingly, these rates are considerably higher compared with the herein observed overall survival rate of $80.6 \%$. This discrepancy might be due to several reasons: first, small patient series are prone to selection bias; indeed, the patient cohorts then were on average younger (median age 55 compared with 60 years). Second, diagnostic criteria have changed from first introduction to the WHO classification in 1993 to the 2000 and 2007 WHO criteria, which have become more objective and reproducible (Kleihues et al, 1993; Kleihues and Cavanee, 2000; Louis et al, 2007). Thus, a caveat remains for atypical meningioma patients diagnosed before 2005. In 2009, Boskos et al (2009) published cause-specific survival data on 19 atypical meningioma patients and found a 5-year survival rate of $80 \%$, which is well in line with the present rate.

On the basis of a single-center series of 248 patients with pituitary adenomas (non-functioning and corticotrophs), an excess mortality of $41 \%$ compared with the general population was reported (Dekkers et al, 2007). Excess mortality was particularly enhanced in corticotroph adenomas (Cushing's disease), especially in patients with persistent Cushing's disease after operation. Even though stratification according to hormone production was not feasible within the present study, our data indicate a trend towards impaired survival of patients with pituitary adenomas. The implicated difference in survival between hormone-producing subtypes remains to be readdressed in further large scale studies. So far, Eurocare data did not list pituitary adenomas among benign CNS tumour types (Sant et al, 2012). Another important issue, which has been raised by Dekkers et al (2007) highlights the considerable neurological comorbidity of patients with pituitary adenoma, which was not only due to local tumour growth but also due to hormonal derangements. Therefore, survival may not be the only accurate outcome measure, but clearly needs to be considered together with quality of life data.

With regard to Schwannoma Eurocare data showed highly favourable outcome with an overall relative survival rate of $96.5 \%$ (98.3\% in Northern Europe) (Sant et al, 2012), which is well in line with our recent findings. However, their data did not allow for further stratification according to tumour location. Within our data set, we were first able to show that patients with acoustic Schwannomas have comparable outcome to those at all other cranial nerves. On the basis of hospital discharge data, McClelland 


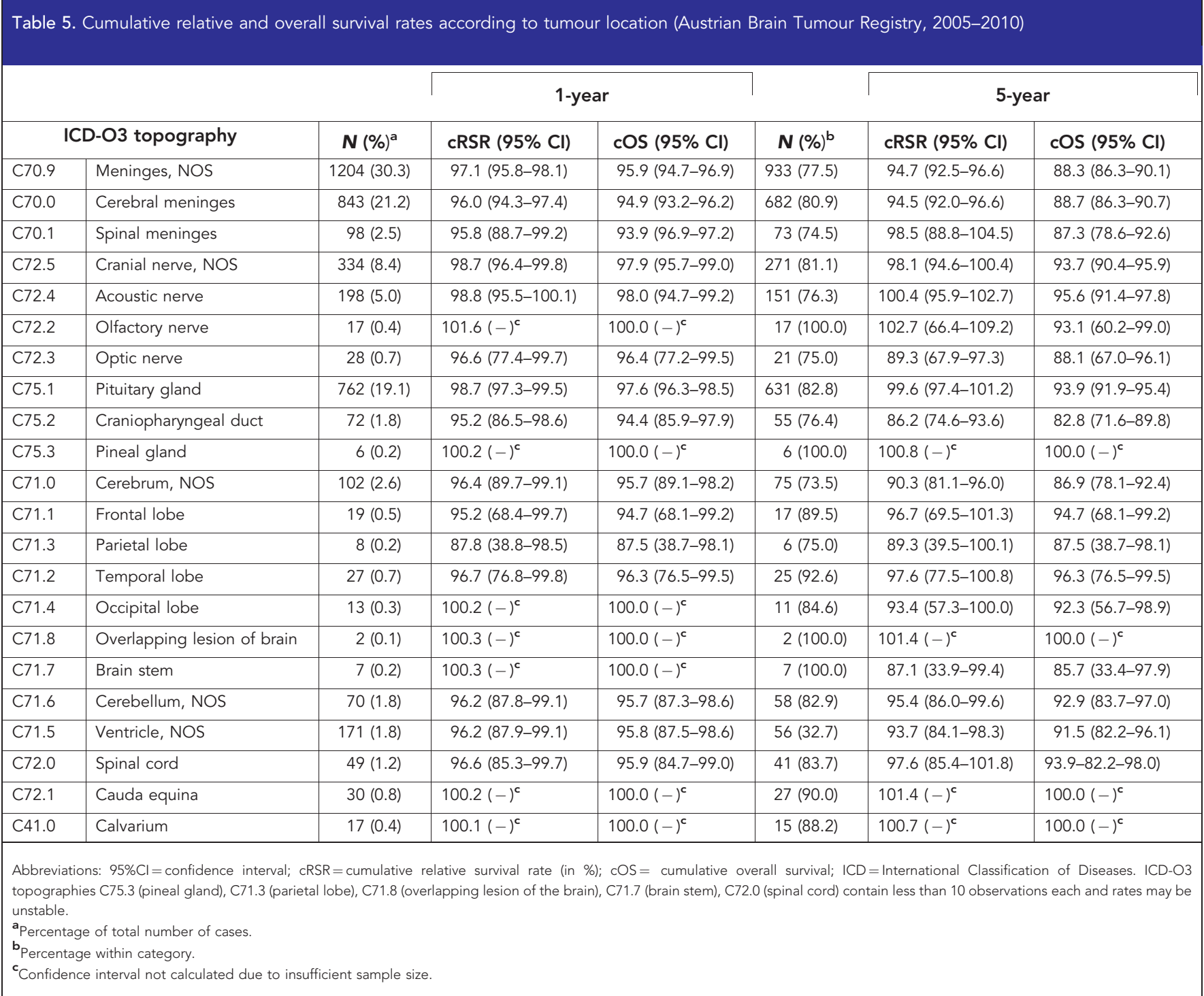

et al (2011) described a postoperative mortality of patients with acoustic Schwannoma in the United States of $0.5 \%(22 / 4886$ patients). Interestingly, mortality was higher in African Americans compared with Caucasians, and lower with high-caseload surgeons, private insurance, younger age, and lower overall morbidity (McClelland et al, 2011). Indeed, these findings on sociodemographic disparities are alerting and deserve further consideration in a European setting.

Pilocytic astrocytomas constitute the only exception, as CBTRUS groups them together with other malignant tumours and routinely reports relative survival rates (Dolecek et al, 2012). According to latest CBTRUS findings, 5-year relative survival from pilocytic astrocytoma is 94.1\% (93.8-95.6), a rate which is similar-even slightly higher compared with the present rate of $92.5 \%$ (Dolecek et al, 2012). In addition, Eurocare data indicate favourable outcome from pilocytic astrocytoma with a 5-year relative survival rate of $97.3 \%$ in those registries, which include also paediatric cases, whereas the overall rate across all age cohorts remained considerably lower with only $80.5 \%$ (Sant et al, 2012). When analysing individual age groups, we observed the same trend with 5-year relative survival of $95.6 \%$ (86.9-98.6\%) in younger patients (0-18 years) but only $74.1 \%$ (27.5-94.6\%) in the age group 45-59 years. However, the relatively small sample sizes per category prevented us from drawing definite conclusions.
This study does have limitations including its retrospective nature as well as its lack of therapy-related data. So far, ABTR constitutes an incidence, mortality and survival database for CNS tumour patients (Wohrer et al, 2009). In the near future, ABTR will transform into a follow-up registry with input of therapyrelated data through members of the Austrian Society of Neurooncology (www.sano.co.at). This will enable assessment of different patterns of care across Austrian neurooncology centers thereby serving as a national and international benchmark system.

In summary, we present relative survival rates of patients with non-malignant CNS tumours in a large contemporary patient cohort. A significant increase in rates indicates improved patient outcome due to advances in the field of neurooncology over the last decades, but underlines the necessity of systematic registration of these types of tumours at the same time. Moreover, relevant issues addressing quality of life, sociodemographic disparities, and differences in patterns of care remain unanswered and need to be addressed in further studies.

\section{ACKNOWLEDGEMENTS}

The ABTR task force (ABTR is a non-for profit organisation) acknowledges the Brain Tumour Epidemiology Consortium 
(BTEC; http://epi.grants.cancer.gov/btec/) as an inspiring scientific forum, fostering the development of multi-center, international and inter-disciplinary collaborations. ABTR looks forward to ongoing collaboration with the Society of Austrian Neurooncology (SANO; http://sano.co.at).

\section{CONFLICT OF INTEREST}

The authors declare no conflict of interest.

\section{REFERENCES}

Bauchet L, Rigau V, Mathieu-Daude H, Figarella-Branger D, Hugues D, Palusseau L, Bauchet F, Fabbro M, Campello C, Capelle L, Durand A, Tretarre B, Frappaz D, Henin D, Menei P, Honnorat J, Segnarbieux F (2007) French brain tumor data bank: methodology and first results on 10,000 cases. J Neurooncol 84: 189-199.

Boskos C, Feuvret L, Noel G, Habrand JL, Pommier P, Alapetite C, Mammar H, Ferrand R, Boisserie G, Mazeron JJ (2009) Combined proton and photon conformal radiotherapy for intracranial atypical and malignant meningioma. Int J Radiat Oncol Biol Phys 75: 399-406.

Burger EH, Groenewald P, Bradshaw D, Ward AM, Yudkin PL, Volmink J (2012) Validation study of cause of death statistics in Cape Town, South Africa, found poor agreement. J Clin Epidemiol 65: 309-316.

Chen CM, Huang AP, Kuo LT, Tu YK (2011) Contemporary surgical outcome for skull base meningiomas. Neurosurg Rev 34: 281-296, discussion 296.

Cho H, Howlader N, Mariotto AB, Cronin KA (2011) Estimating relative survival for cancer patients from the SEER Program using expected rates based on Ederer I versus Ederer II method. Surveillance Research Program. NCI Technical Report \#2011-01. Available at: www.surveillance. cancer.gov/reports/.

Cook MB, Dawsey SM, Freedman ND, Inskip PD, Wichner SM, Quraishi SM, Devesa SS, McGlynn KA (2009) Sex disparities in cancer incidence by period and age. Cancer Epidemiol Biomarkers Prev 18: 1174-1182.

Cornwell P, Dicks B, Fleming J, Haines TP, Olson S (2012) Care and support needs of patients and carers early post-discharge following treatment for non-malignant brain tumour: establishing a new reality. Support Care Cancer 20: 2595-2610.

Coskun M, Straube W, Hurkmans CW, Melidis C, De Haan PF, Villa S, Collette S, Weber DC (2013) Quality assurance of radiotherapy in the ongoing EORTC 22042-26042 trial for atypical and malignant meningioma: results from the dummy runs and prospective individual case Reviews. Radiat Oncol 8: 23.

Dekkers OM, Biermasz NR, Pereira AM, Roelfsema F, van Aken MO, Voormolen JH, Romijn JA (2007) Mortality in patients treated for Cushing's disease is increased, compared with patients treated for nonfunctioning pituitary macroadenoma. J Clin Endocrinol Metab 92: 976-981.

Dolecek TA, Propp JM, Stroup NE, Kruchko C (2012) CBTRUS Statistical Report: Primary Brain and Central Nervous System Tumors Diagnosed in the United States in 2005-2009. Neuro-Oncol 14(Suppl 5): v1-v49.

Ederer F, Axtell LM, Cutler SJ (1961) The relative survival rate: a statistical methodology. Natl Cancer Inst Monogr 6: 101-121.

Ederer F, Heise H (1959) Instructions to IBM 650 Programmers in Processing Survival Computations, Technical, End Results Evaluation Section. National Cancer Institute, Methodological note No 10.

Evans DG, Birch JM, Ramsden RT, Sharif S, Baser ME (2006) Malignant transformation and new primary tumours after therapeutic radiation for benign disease: substantial risks in certain tumour prone syndromes. J Med Genet 43: 289-294.

Fritz A, Percy C, Jack A, Shanmugaratnam K, Sobin L, Parkin M, Whelan S (eds) (2000) International classification of diseases for oncology, 3rd edn. World Health Organization: Geneva, Switzerland.

Gnekow AK, Falkenstein F, von Hornstein S, Zwiener I, Berkefeld S, Bison B, Warmuth-Metz M, Driever PH, Soerensen N, Kortmann RD, Pietsch T, Faldum A (2012) Long-term follow-up of the multicenter, multidisciplinary treatment study HIT-LGG-1996 for low-grade glioma in children and adolescents of the German Speaking Society of Pediatric Oncology and Hematology. Neuro Oncol 14: 1265-1284.
Goyal LK, Suh JH, Mohan DS, Prayson RA, Lee J, Barnett GH (2000) Local control and overall survival in atypical meningioma: a retrospective study. Int J Radiat Oncol Biol Phys 46: 57-61.

Hu CY, Xing Y, Cormier JN, Chang GJ (2013) Assessing the utility of cancerregistry-processed cause of death in calculating cancer-specific survival. Cancer 119: 1900-1907.

Israel National Cancer Registry (2013), http://www.health.gov.il/English/ MinistryUnits/HealthDivision/Icdc/Icrc/Pages/default.aspx, last assessed 03/07/2013.

Klaeboe L, Lonn S, Scheie D, Auvinen A, Christensen HC, Feychting M, Johansen C, Salminen T, Tynes T (2005) Incidence of intracranial meningiomas in Denmark, Finland, Norway and Sweden, 1968-1997. Int J Cancer 117: 996-1001.

Kleihues P, Burger PC, Scheithauer BW (1993) The new WHO classification of brain tumours. Brain Pathol 3: 255-268.

Kleihues P, Cavanee WK (eds) (2000) Pathology and Genetics of Tumours of the Nervous System. IARC Press: Lyon, France.

Kuhnt D, Bauer MH, Ganslandt O, Nimsky C (2013) Functional imaging: where do we go from here? J Neurosurg Sci 57: 1-11.

Kuhnt D, Bauer MH, Nimsky C (2012) Brain shift compensation and neurosurgical image fusion using intraoperative MRI: current status and future challenges. Crit Rev Biomed Eng 40: 175-185.

Lafrance JP, Rahme E, Iqbal S, Leblanc M, Pichette V, Elftouh N, Vallee M (2013) Magnitude of discordance between registry data and death certificate when evaluating leading causes of death in dialysis patients. BMC Med Res Methodol 13: 51.

Legler JM, Ries LA, Smith MA, Warren JL, Heineman EF, Kaplan RS, Linet MS (1999) Cancer surveillance series [corrected]: brain and other central nervous system cancers: recent trends in incidence and mortality. J Natl Cancer Inst 91: 1382-1390.

Louis DN, Ohgaki H, Wiestler D, Cavanee WK (eds) (2007) WHO Classification of Tumours of the Central Nervous System. IARC Press: Lyon, France.

McCarthy BJ, Kruchko C, Dolecek TA (2013) The Impact of the Benign Brain Tumor Cancer Registries Amendment Act (Public Law 107-260) on Non-malignant Brain and Central Nervous System Tumor Incidence Trends. J Registry Manag 40: 32-35.

McCarthy BJ, Schellinger KA, Propp JM, Kruchko C, Malmer B (2009) A case for the worldwide collection of primary benign brain tumors. Neuroepidemiology 33: 268-275.

McClelland 3rd S, Guo H, Okuyemi KS (2011) Morbidity and mortality following acoustic neuroma excision in the United States: analysis of racial disparities during a decade in the radiosurgery era. Neuro Oncol 13: $1252-1259$.

Palma L, Celli P, Franco C, Cervoni L, Cantore G (1997) Long-term prognosis for atypical and malignant meningiomas: a study of 71 surgical cases. J Neurosurg 86: 793-800.

Perry A, Gutmann DH, Reifenberger G (2004) Molecular pathogenesis of meningiomas. J Neurooncol 70: 183-202.

Sant M, Minicozzi P, Lagorio S, Borge Johannesen T, Marcos-Gragera R, Francisci S (2012) Survival of European patients with central nervous system tumors. Int J Cancer 131: 173-185.

Shibui S (2012) [The present status and trend of brain tumors based on the data of the Brain Tumor Registry of Japan]. Brain Nerve 64: 286-290.

Solheim O, Jakola AS, Gulati S, Johannesen TB (2012) Incidence and causes of perioperative mortality after primary surgery for intracranial tumors: a national, population-based study. J Neurosurg 116: 825-834.

Sutra S, Chirawatkul A, Leelapanmetha P, Sirisuwan S, Thepsuthammarat K (2012) Evaluation of causes-of-death: which statistics should we rely on, hospital deaths or vital statistics? J Med Assoc Thai 95(Suppl 7): S262-S273.

Talback M, Stenbeck M, Rosen M (2004) Up-to-date long-term survival of cancer patients: an evaluation of period analysis on Swedish Cancer Registry data. Eur J Cancer 40: 1361-1372.

van Alkemade H, De Leau M, Dieleman EM, Kardaun JW, van Os R, Vandertop WP, van Furth WR, Stalpers LJ (2012) Impaired survival and long-term neurological problems in benign meningioma. Neuro Oncol 14: 658-666.

Vroonen L, Jaffrain-Rea ML, Petrossians P, Tamagno G, Chanson P, Vilar L, Borson-Chazot F, Naves LA, Brue T, Gatta B, Delemer B, Ciccarelli E, Beck-Peccoz P, Caron P, Daly AF, Beckers A (2012) Prolactinomas resistant to standard doses of cabergoline: a multicenter study of 92 patients. Eur J Endocrinol 167: 651-662. 
Woehrer A (2013) Brain tumor epidemiology in Austria and the Austrian Brain Tumor Registry. Clin Neuropathol 32: 269-285.

Wohrer A, Waldhor T, Heinzl H, Hackl M, Feichtinger J, Gruber-Mosenbacher U, Kiefer A, Maier H, Motz R, Reiner-Concin A, Richling B, Idriceanu C, Scarpatetti M, Sedivy R, Bankl HC, Stiglbauer W, Preusser M, Rossler K, Hainfellner JA (2009) The Austrian Brain Tumour Registry: a cooperative way to establish a population-based brain tumour registry. J Neurooncol 95 : 401-411.

Wong JM, Panchmatia JR, Ziewacz JE, Bader AM, Dunn IF, Laws ER, Gawande AA (2012) Patterns in neurosurgical adverse events: intracranial neoplasm surgery. Neurosurg Focus 33: E16.
Zachenhofer I, Wolfsberger S, Aichholzer M, Bertalanffy A, Roessler K, Kitz K, Knosp E (2006) Gamma-knife radiosurgery for cranial base meningiomas: experience of tumor control, clinical course, and morbidity in a follow-up of more than 8 years. Neurosurgery 58 : 28-36. discussion 28-36.

This work is published under the standard license to publish agreement. After 12 months the work will become freely available and the license terms will switch to a Creative Commons AttributionNonCommercial-Share Alike 3.0 Unported License. 